\title{
REVUE
}

\section{L'AUGMENTATION DES PROPRIÉTÉS ANTIRACHITIQUES DU LAIT PAR LES RADIATIONS LUIMINEUSES}

\author{
par \\ G. GÉNIN \\ Ingénieur E. P. C.
}

Depuis quelques années, l'irradiation du lait en vue d'augmenter ses qualités antirachitiques est devenue une technique très employée et dont le principe essentiel consiste à exposer le lait à l'action de radiations d'une certaine qualité et d'une certaine intensité. Pour augmenter l'activité antirachitique du lait, il y a avantage à utiliser de préférence les radiations ultra-violettes et plus spécialement celles dont la longueur d'onde est comprise entre 2.300 et $3.130 \AA$. Malheureusement, il est matériellement impossible de produire des radiations dont la longueur d'onde soit rigoureusement comprise dans les limites précédentes, sans en même temps donner naissance à des radiations de plus grande longueur d'onde et même à des radiations lumineuses. De même, il n'est pas possible d'éliminer efficacement ces radiations inutiles par un écran approprié, car tout écran de ce genre est généralement trop absorbant pour les radiations utiles.

Or, l'énergie lumineuse, dans certaines conditions, peut entraîner l'apparition d'un goût défectueux du lait. Ce point a été démontré par de nombreux auteurs comme Hammer et Cordes, Tracy et RUEHE qui ont montré que le lait acquiert un goût défectueux lorsqu'il est exposé aux rayons solaires. Il en résulte que dans ces conditions, l'irradiation du lait qui consiste industriellement à le soumettre à l'action de rayons ultra-violets, de rayons lumineux visibles et de rayons infra-rouges, risque, si l'opération est conduite d'une façon imparfaite, de faire apparaître dans le lait des goûts défectueux qui en interdisent la consommation. Il faut d'ailleurs rapprocher cet inconvénient d'un autre analogue qui a retardé pendant longtemps les applications de la pasteurisation du lait et qui se manifestait par l'apparition d'un goût de cuit. Il est certain que l'irradiation du lait n'a pas encore eu toutes les applications que cette technique mérite, mais il faut espérer que les progrès apportés à la technique de l'irradiation permettront de faire disparaître l'inconvénient que nous venons de signaler comme les progrès réalisés dans la pasteurisation ont permis d'éliminer, sinon entièrement, du moins en grande partie, le goût de cuit du lait. 
C'est pour cette raison qu'il nous a paru intéressant de rendre compte d'une étude entreprise à l'Université de Wisconsin par K. G. Weckel, H. C. Jackson, R. Haman et H. Stennbock, qui ont cherché à déterminer quels sont les facteurs qui entrainent l'apparition d'un goût défectueux du lait, lorsque celui-ci est irradié en vue d'augmenter ses propriétés antirachitiques.

Il est d'ailleurs assez difficile de distinguer le goût particulier qui provient de l'irradiation du lait d'autres goûts défectueux dus à des facteurs étrangers. L'irradiation du lait a surtout pour conséquence de faire apparaître un goût de brûlé, mais qui, si l'irradiation est prolongée, peut évoluer et se transformer et donner ce qu'on appelle parfois un goût de champignon au lait. Il faut distinguer ce goût du goût de papier ou de carton qui peut résulter de l'action sur certains constituants du lait soit du métal dans lequel il est contenu, soit de certaines radiations particulières. Il faut également ne pas le confondre avec le goût qui apparaît lorsque le lait est soumis à l'action de l'ozone et qui est particulièrement désagréable. Le goût particulier dû à l'irradiation est un goût que l'on a défini du nom " d'activé ". Il semble entraîner surtout des transformations dans la fraction protéinique du lait, et il ne se confond pas avec le goût de cuit du lait qui apparaît lorsque celui-ci a été soumis à l'action de la chaleur.

Les expériences que nous résumons ont été effectuées en utilisant pour irradier le lait soit une lampe à arc de carbone, soit une lampe de quartz à vapeur de mercure. On utilisait des électrodes de carbone, fournies par des fabricants différents et on déterminait avec soin la composition du spectre émis par chacune de ces lampes. Dans certains cas, on faisait appel à des prismes ou à des filtres, afin de ne faire agir sur le lait que des longueurs d'onde comprises entre des limites bien déterminées.

Une conclusion générale résulte d'abord de ces études, c'est qu'il apparaît une saveur particulière lorsque le lait est irradié pendant un temps suffisant, quelle que soit la nature : quartz ou verre, de la lampe. On a done pu en conclure, connaissant les qualités de transmission de la lumière du verre ou du quartz et ayant établi d'autre part que seules les radiations visibles et les radiations infra-rouges ne produisent pas d'action sur le lait, que l'apparition du goût défectueux doit être due à l'action de longueurs d'onde inférieures à $7.000 \AA$.

On a alors examiné deux groupes de radiations : les unes à longueur d'onde comprise entre 2.300 et $3.100 \AA$ et les autres comprises entre 3.100 et $7.000 \AA$. Ces essais ont montré que dans presque tous les cas, l'apparition de la saveur défectueuse est due à l'action de radiations ayant une longueur d'onde inférieure à $3.100 \AA$, c'est- 
à-dire aux longueurs d'onde qui sont réputées comme étant les plus: efficaces pour développer les qualités antirachitiques du lait.

On a alors approfondi la question et examiné les différentes: longueurs d'onde comprises dans les limites 2.300 et 3.100 ou comprises entre les limites 3.100 et $7.000 \AA$. Les résultats de cette nouvelle étude ont montré que les radiations de longueurs d'onde comprises entre 2.600 et $3.100 \AA$ favorisent moins l'apparition du. goût défectueux que les radiations de longueurs d'onde inférieures à $2.600 \mathrm{~A}^{\circ}$. De même dans l'autre groupe de radiations, ce sont celles: de longueurs d'onde comprises entre 3.100 et $3.800 \AA$ qui sont beaucoup plus dangereuses que celles dont la longueur d'onde est supérieure à $3.800 \AA$.

On voit done par ces résultats qu'un pas important a été fait dans la connaissance des phénomènes qui entourent l'irradiation du lait. Certes, il reste encore beaucoup à faire, puisqu'on ne sait pas. réaliser des sources de radiations ne donnant que des radiations. comprises entre des limites parfaitement déterminées, mais la question est néanmoins en bonne voie, puisqu'on connaît désormais: les radiations les plus utiles et au contraire celles qui sont nuisibles: et qu'il y a intérêt à supprimer.

\section{BIBLIOGRAPHIE ANALYTIQUE}

\section{LES LIVRES}

Orla-Jensen (S.), Otte (N. C.) et Snog-KJaer (Agnete). - The vitamin and nitrogen requirements of the lactic acid bacteria (Exigences en vitamines et en azote des bactéries lactiques). Mémoires de l'Académie Royale des Sciences et des Lettres de Danemark, Copenhague. Section des Sciences, $9^{\mathrm{e}}$ série, t. VI, no 5. Copenhague : Levin et Munksgaard, Norregade, 6, Copenhague. 1 brochure de 52 pages, 1936. Prix : 3 cour. 75.

I. Exigences des bactéries lactiques en vitamines.

Les véritables bactéries lactiques exigent une substance thermostable alcaline. Celle-ci est absorbée par du charbon activé à toutes les concentrations en ions $\mathrm{H}$ dans la série acide, et, commə la lactoflavine, elle est facilement éliminée par lavage à l'aide de la pyridine et de l'alcool méthylique. Cette substance a une influence favorable sur la croissance de la levure, et peut donc être considérée comme identique à l'acide pantothénique, qui est le constituant principal du bios. De nombreux faits semblent indiquer que cet activateur intervenant dans le lait est aussi identique à $\mathrm{B} 6$, ce qui signifie que la vitamine B 2, doit être constituée de bios et de lactoflavine.

Les bactéries lactiques exigent la lactoflavine en quantités variables en 